\title{
Trattamento emodialitico dell'insufficienza renale acuta
}

\author{
C. Meloni ${ }^{1}$, M. Morosetti ${ }^{2}$, L. Meschini ${ }^{3}$, G. Palombo ${ }^{2}$, C. Galderisi ${ }^{2}$, M. Taccone-Gallucci ${ }^{2}$, \\ S. Di Giulio ${ }^{3}$, C.U. Casciani ${ }^{2}$
}

\author{
${ }^{1}$ Istituto Tipizzazione Tissutale, CNR, L'Aquila \\ ${ }^{2}$ Clinica Chirurgica, Università Tor Vergata, Roma \\ ${ }^{3}$ Nefrologia e Dialisi, Ospedale GB Grassi, USL Roma D
}

\section{A}

nche se recentemente sono stati descritti miglioramenti nella prognosi dei pazienti affetti da insufficienza renale acuta (IRA) (1), tale sindrome presenta ancora oggi una prognosi sfavorevole specialmente nel caso in cui compaia in corso di Multiple Organ Failure (MOF) (2). Peraltro, il trattamento emodialitico dell'IRA richiede spesso particolari accorgimenti tecnici a causa delle condizioni generali, spesso critiche, di questi pazienti (3). Le caratteristiche del trattamento sostitutivo ideale nei pazienti affetti da IRA sono riportate in Tabella I. La scelta della metodica dialitica, pertanto, deve essere eseguita in relazione alle necessità individuali del paziente e alle caratteristiche delle tecniche disponibili (3). Tali considerazioni comprendono il tipo di accesso vascolare, il rischio di sanguinamento, le condizioni emodinamiche e la necessità di rimuovere un eccesso di liquidi (4).

Scopo del nostro lavoro è stato quello di verificare l'efficacia clinica e la tollerabilitià di diverse metodiche emodialitiche in corso di IRA non complicata o in corso di MOF.

\section{Pazienti e metodi}

Dal 01.01.1988 al 31.10.96 sono venuti alla nostra osservazione 198 pazienti
(118 maschi, 80 femmine, età media $50.3 \pm 18.8$ anni, range 18 - 81) affetti da IRA in cui era necessario un trattamento dialitico. I pazienti sono stati divisi in 2 gruppi a seconda dell'assenza (Gruppo I) o della presenza (Gruppo II) di MOF.

Gruppo I: 88 pazienti (55 maschi, 33 femmine, eta media $48.2 \pm 15.5$ anni, range 18 - 72) affetti da IRA "isolata". Le cause di IRA sono riportate in Tabella II. Cinquantaquattro pazienti erano oligoanurici (diuresi/24 h media $170.9 \pm$ $30 \mathrm{cc}$ ), mentre 34 casi presentavano una diuresi residua di $1339 \pm 331 \mathrm{cc} / 24 \mathrm{~h}$. Nessun paziente presentava MOF, in nessun caso era necessaria una ventilazione meccanica.

Quarantuno pazienti (26 maschi, 15 femmine, età media $47.0 \pm 16.3$ anni, range 20-72 ) venivano trattati con acetate free biofiltration (AFB), secondo caratteristiche precedentemente descritte
(5). Venivano eseguite in totale 732 sedute con un volume medio reinfuso di $1.86 \pm 0.32 \mathrm{~L} / \mathrm{h}$ (range 1.2 - 3.5). L'ultrafiltrazione veniva impostata di volta in volta a seconda delle necessità del paziente; il calo ponderale medio per la rimozione di liquidi era di $3.0 \pm 1.7 \mathrm{~kg}$ (range 1.0 - 5.6). La durata del trattamento era di 180 minuti, 4 volte/settimana per un periodo medio di $29.7 \pm$ 15.3 giorni (range 11 - 47 giorni ).

In quarantasette pazienti (29 maschi, 18 femmine, $50.0 \pm 15.6$ anni, range 18 81) venivano eseguite 803 sedute di bicarbonato dialisi della durata di $180 \mathrm{mi}-$ nuti, 4 volte/settimana per un periodo medio di $28.0 \pm 13.3$ giorni (range 13 37). La rimozione media di liquidi era di $2.75 \pm 1.6 \mathrm{~kg} /$ seduta (range $1.0-5.5$ )

Tutte le sedute dialitiche venivano effettuate con membrane in Poliacrilonitrile (PAN) a fibre cave di varia superficie

\section{TAB. I - CARATTERISTICHE DEL TRATTAMENTO SOSTITUTIVO IDEALE IN PAZIENTI AFFETTI DA IRA}

- Adeguatezza dialitica in relazione al Protein Catabolic Rate (PCR);

- Adeguata correzione dell'acidosi metabolica e del bilancio elettrolitico;

- Ottimale tolleranza dialitica;

- Stabilità cardiovascolare durante la rimozione dei liquidi in eccesso;

- Bassa dose o non uso di anticoagulanti. 
TAB. II - CAUSE DI IRA “NON COMPLICATA" IN 88 PAZIENTI (GRUPPO I)

\begin{tabular}{lcc}
\hline Diagnosi & $\mathbf{N}^{\circ}$ casi & $\%$ \\
\hline IRA post-chirurgica & 49 & 55.6 \\
IRA da farmaci & 17 & 19.3 \\
Crush syndrome & 9 & 10.2 \\
Ustioni estese & 3 & 3.4 \\
Sindrome emolitico-uremica & 2 & 2.9 \\
Cause incerte & 8 & 9.1 \\
\hline
\end{tabular}

$\left(1.21 .6 \mathrm{~m}^{2}\right)$. La temperatura media del dialisato era di $36.7 \pm 0.3^{\circ} \mathrm{C}$, la conducività media era di $14.5 \pm 0.5 \mathrm{mS}$. Come accesso vascolare veniva utilizzato un catetere bilume posizionato in una vena (femorale o succlavia) profonda per via percutanea, con un flusso ematico medio di $275 \pm 59 \mathrm{ml} / \mathrm{min}$ (range $220-350$ ). Veniva eseguita un'emogasanalisi pre e post-dialisi e, durante la prima settimana, ogni ora di trattamento; BUN, creatinina, sodio, potassio, calcio, fosforo, ematocrito, emoglobina, venivano valutati prima e dopo ogni trattamento dialitico. Il $\mathrm{Kt} / \mathrm{V}$ veniva calcolato secondo una formula di Gotch modificata (6). La pressione arteriosa e la frequenza cardiaca venivano monitorizzate in continuo (monitor Hewlett Packard 78354A) durante ogni trattamento. Sintomi intradialitici come ipotensione, vomito, cefalea, crampi venivano registrati durante i trattamenti. Se uno o più sintomi erano presenti la dialisi veniva descritta come sintomatica.

Gruppo II: 110 pazienti (63 maschi, 47 femmine, età media $52.6 \pm 15.2$ anni, range 24 - 81) affetti da IRA in MOF venivano trattati con dialisi. Le malattie responsabili di IRA in questo gruppo sono riportate in Tabella III. MOF era presente in tutti i casi: 81 pazienti $(73.6 \%)$ presentavano insufficienza respiratoria ed erano assistiti meccanicamente; in $72 \mathrm{ca}$ si $(65.4 \%)$ il circolo veniva supportato mediante infusione continua di catecolamine a dosaggi elevati; 19 (11.8\%) pazienti presentavano un'insufficienza epatica grave, mentre in 28 casi ( $25.4 \%$ ) vi erano difetti gravi della coagulazione o sanguinamento in atto: questi pazienti venivano trattati con bicarbonato dialisi giornaliera a ricircolo (Daily-HD) senza anticoagulanti. Dei 110 pazienti, 35 venivano sottoposti a Daily-HD, 67 pazienti erano trattati con emofiltrazione artero-venosa continua (CAVH) e 8 casi ve- nivano trattati con emofiltrazione venovenosa continua ( $\mathrm{CVVH})$.

L' APACHE II score (7) era calcolato in tutti i pazienti ed era rispettivamente di $28.0 \pm 4.9$ (range 18-47) nel gruppo trattato con CAVH, di $28.6 \pm 6.0$ (range 2144) nel gruppo trattato con Daily-HD e di $29.1 \pm 5.6$ (range 18-45) nel gruppo trattato con CVVH D. La pressione arteriosa media (MAP), monitorizzata intraarteriosamente (monitor Siemens Sirecust 402 ) era di $77.9 \pm 8.6 \mathrm{mmHg}$, la frequenza cardiaca (HR) di $96.5 \pm 17.3$ battiti/min. In $74(67.2 \%)$ casi era in atto una nutrizione parenterale totale, in 19 casi $(17.2 \%)$ veniva utilizzata una nutrizione enterale, mentre in 12 casi $(10.9 \%)$ veniva prescritta un'alimentazione orale arricchita con integratori calorici.

Quattrocentosettantanove trattamenti con Daily-HD venivano eseguiti in $35 \mathrm{pa}-$ zienti (20 maschi, 15 femmine, età media $49.7 \pm 11.0$ anni, range $24-76$ ) con un macchina portatile, ricircolante $25 \mathrm{li}$ tri di bagno ad alto flusso, in accordo con la metodica di Buoncristiani (8). La composizione del dialisato veniva modificata a seconda delle necessità cliniche del singolo paziente. In tutti i casi veniva utilizzata una membrana in PAN di varia superficie $\left(1.0-1.6 \mathrm{~m}^{2}\right)$ con un flusso ematico medio di $215 \mathrm{ml} / \mathrm{min}$ (range 140
- 350) ottenuto mediante catetere in vena profonda. Tutti i pazienti sono stati trattati quoditianamente per un periodo medio di 90 minuti (range 75 - 120). La durata media del trattamento è stata di 17.0 \pm 5.8 giorni (range 7 - 31 ).

Sessantasette pazienti (39 maschi, 28 femmine, età media $52.8 \pm 18.9$ anni, range 27 - 78) venivano trattati con CAVH. Venivano utilizzati filtri in PAN (superficie 0.43 e $0.80 \mathrm{~m}^{2}$ ) connessi a due cateteri a lume singolo posizionati in arteria e vena femorale. I filtri venivano cambiati a causa di parziale o totale coagulazione del circuito o in presenza di una funzione inadeguata (ultrafiltrato $<250 \mathrm{ml} / \mathrm{h}$ per 3 ore consecutive). In media venivano utilizzati $3.5 \pm 1.6$ filtri / paziente, con una vita media di 58.2 ore per filtro. Pazienti ipercatabolici ricevevano una emodiafiltrazione (CAVHD), con dialisato ad un flusso di $1 \mathrm{~L} / \mathrm{h}$.

L' emofiltrazione veno-venosa continua (CVVH) veniva eseguita in 8 pazienti (4 maschi, 4 femmine, età media 53.0 i 12.6 anni, range 33 - 63 anni) sottoposti a trapianto di fegato con IRA. La metodica veniva eseguita con accorgimenti tecnici in accordo con altri Autori (9). La pompa ematica a flusso di $100 \mathrm{ml} / \mathrm{min}$, mediante una cannula bilume posizionata in vena femorale, ed apparecchiatura Gambro AK10 con apposito softwear. In tutti casi veniva utilizzata membrana in PAN (superficie di $0.80 \mathrm{~m}^{2}$ ). In 6 casi veniva inserito un flusso di dialisato (CVVHD) a $1000 \mathrm{cc} / \mathrm{h}$. In media venivano utilizzati $4.0 \pm 1.3$ filtri / paziente, con una vita media di 44.7 ore per filtro.

BUN, creatinina, sodio, potassio, calcio, fosforo, ematocrito, emoglobina, venivano valutati prima e dopo ogni DailyHD ed ogni 24 ore di trattamento con CAVH e CVVH.

\section{TAB. III - CAUSE DI IRA IN CORSO DI MOF IN 110 PAZIENTI (GRUPPO II)}

\begin{tabular}{lcc}
\hline Diagnosi & $\mathbf{N}^{\circ}$ casi & $\%$ \\
\hline IRA post-chirurgica & 31 & 28.1 \\
Sepsi & 27 & 24.5 \\
Ustioni estese & 16 & 14.5 \\
Crush syndrome & 12 & 10.9 \\
Neoplasie ematologiche & 10 & 9.1 \\
Insufficienza epatica grave & 8 & 7.2 \\
IRA post partum/dic & 3 & 2.7 \\
IRA da farmaci & 2 & 1.8 \\
Causa imprecisata & 1 & 0.9 \\
\hline
\end{tabular}


I dati sono espressi come media \pm deviazione standard. L'analisi statistica è stata eseguita mediante uno Student t-test; un $\mathrm{p}<0.05$ è stato considerato significativo.

\section{Risultati}

In Tabella IV sono riportati i risultati relativi al trattamento con AFB e bicarbonato dialisi standard che mostrano come l'efficacia dialitica, la rimozione di liquidi, il bilancio elettrolitico ed emogasanalitico, la durata del trattamento siano sovrapponibili. Al contrario, una differenza statisticamente significativa era presente nell'incidenza di dialisi sintomatiche tra le 2 metodiche $(5.3 \%$ durante AFB versus $11.9 \%$ in bicarbonato dialisi standard, $\mathrm{p}<0.01)$. In particolare gli episodi ipotensivi avevano un'incidenza maggiore durante bicarbonato dialisi nei confronti del trattamento con AFB (rispettivamente 9.8 versus $3.2 \%, \mathrm{p}<0.01$ ). La sopravvivenza media era di $80.5 \%$ senza differenze statisticamente significative tra i 2 tipi di trattamento $(80.4 \%$ e $80.8 \%$ rispettivamente).

In Tabella $\mathrm{V}$ sono riportati i dati relativi al trattamento dialitico del Gruppo II. Tutte le metodiche risultano adeguate per la rimozione di liquidi, bilancio elettrolitico e tolleranza dialitica, nonostante le precarie condizioni cliniche di questi pazienti. Infatti MAP e HR non si modificano al termine del trattamento rispetto all' inizio, anche se durante 61 (12.7\%) sedute di Daily-HD era necessario ricorrere all'infusione di soluzione fisiologica o macromolecole. L'uso di CAVH ha permesso il controllo dei livelli di azotemia. In 24 casi $(35.8 \%)$ uno stato ipercatabolico richiedeva l'uso di una CAVHD che permetteva in tutti $\mathrm{i}$ casi un buon controllo metabolico. La tecnica di Daily-HD al contrario otteneva un controllo dei livelli di azotemia meno soddisfacenti con una diminuzione media del $23 \%$ durante ogni trattamento. La CVVH richiedeva per lo stato ipercatabolico dei pazienti l'uso del dialisato (CVVHD) nel 75\% dei casi.

Infine, la sopravvivenza media era del $35.4 \%$ senza differenze statisticamente significative tra i 3 gruppi (rispettivamente $35.8 \%$ nel gruppo trattato con CAVH, 34.2\% nel gruppo trattato con Daily-HD e $37.5 \%$ in quelli trattati con CVVH).

TAB. IV - DATI EMATOCHIMICI E CLINICI DI 88 PAZIENTI (GRUPPO I) AFFETTI DA IRA DOPO TRATTAMENTO CON AFB E $\mathrm{HCO}_{3}$-HD

\begin{tabular}{llcc}
\hline & & $\mathbf{4 1} \mathbf{~ A F B}$ & $\mathbf{4 7} \mathbf{H C O}_{3}$-HD \\
\hline BUN & $(\mathrm{mg} / \mathrm{dl})$ & $47.6 \pm 11.1$ & $45.0 \pm 18.3$ \\
Creatinina & $(\mathrm{mg} / \mathrm{dl})$ & $3.2 \pm 1.4$ & $3.4 \pm 1.2$ \\
Sodio & $(\mathrm{mEq} / \mathrm{L})$ & $144 \pm 5.6$ & $146 \pm 5.9$ \\
Potassio & $(\mathrm{mEq} / \mathrm{L})$ & $3.7 \pm 0.4$ & $4.0 \pm 0.9$ \\
Calcio & $(\mathrm{mg} / \mathrm{dl})$ & $9.6 \pm 1.5$ & $9.6 \pm 0.8$ \\
Fosforo & $(\mathrm{mg} / \mathrm{dl})$ & $4.3 \pm 1.3$ & $4.0 \pm 1.1$ \\
$\mathrm{Ht}$ & $(\%)$ & $33.4 \pm 4.1$ & $33.0 \pm 3.2$ \\
$\mathrm{Hb}$ & $(\mathrm{g} / \mathrm{L})$ & $11.7 \pm 1.9$ & $11.2 \pm 2.7$ \\
$\mathrm{pH}$ & & $7.44 \pm 0.03$ & $7.42 \pm 0.04$ \\
$\mathrm{HCO}$ & & $24.6 \pm 1.7$ & $24.0 \pm 1.1$ \\
$\mathrm{MAP}$ & $(\mathrm{mmol} / \mathrm{L})$ & $106 \pm 15.5$ & $105 \pm 11.8$ \\
$\mathrm{HR}$ & $(\mathrm{mmHg})$ & $81 \pm 18$ & $89 \pm 16$ \\
$\mathrm{Kt} / \mathrm{V}$ & $(\mathrm{b} / \mathrm{min})$ & $1.1 \pm 0.05$ & $1.0 \pm 0.04$ \\
Dialisi sintomatiche & $(\%)$ & 4.8 & 11.0 \\
Delta peso & $(\mathrm{kg} / \mathrm{h})$ & $1.3 \pm 0.3$ & $1.5 \pm 0.4$ \\
\hline
\end{tabular}

TAB. V - DATI EMATOCHIMICI E PARAMETRI CLINICI DI 110 PAZIENTI (GRUPPO II) AFFETTI DA IRA IN CORSO DI MOF DOPO TRATTAMENTO CON CAVH, DIALISI GIORNALIERA E CVVH

\begin{tabular}{llccc}
\hline $\mathbf{N}^{\circ}$ pazienti & 67 CAVH & 35 DAILY-HD & 8 CVVH \\
\hline BUN & $(\mathrm{mg} / \mathrm{dl})$ & $77.0 \pm 25.3$ & $88.0 \pm 13.1$ & $90.3 \pm 16.3$ \\
Creatinina & $(\mathrm{mg} / \mathrm{dl})$ & $3.9 \pm 1.6$ & $4.6 \pm 1.3$ & $5.0 \pm 1.3$ \\
Sodio & $(\mathrm{mEq} / \mathrm{L})$ & $143 \pm 7.2$ & $138 \pm 4.6$ & $141 \pm 5.0$ \\
Potassio & $(\mathrm{mEq} / \mathrm{L})$ & $4.2 \pm 0.7$ & $4.0 \pm 0.4$ & $3.9 \pm 0.7$ \\
Calcio & $(\mathrm{mg} / \mathrm{dl})$ & $9.5 \pm 1.1$ & $8.9 \pm 1.1$ & $8.2 \pm 0.7$ \\
Fosforo & $(\mathrm{mg} / \mathrm{dl})$ & $5.4 \pm 1.4$ & $5.5 \pm 1.3$ & $4.7 \pm 1.2$ \\
$\mathrm{Ht}$ & $(\%)$ & $30.6 \pm 4.1$ & $32.7 \pm 4.1$ & $31.4 \pm 3.6$ \\
$\mathrm{Hb}$ & $(\mathrm{g} / \mathrm{L})$ & $10.1 \pm 2.8$ & $10.9 \pm 2.2$ & $10.6 \pm 1.7$ \\
$\mathrm{pH}$ & & $7.43 \pm 0.04$ & $7.44 \pm 0.03$ & $7.44 \pm 0.04$ \\
$\mathrm{HCO}^{-}$ & $(\mathrm{mmol} / \mathrm{L})$ & $23.4 \pm 2.0$ & $23.7 \pm 0.8$ & $24.1 \pm 0.6$ \\
$\mathrm{MAP}^{3}$ & $(\mathrm{mmHg})$ & $72.5 \pm 5.9$ & $72.5 \pm 4.0$ & $73.1 \pm 6.6$ \\
$\mathrm{HR}^{2}$ & $(\mathrm{~b} / \mathrm{min})$ & $89 \pm 11$ & $91 \pm 15$ & $88 \pm 17$ \\
Delta peso & $(\mathrm{kg} / \mathrm{h})$ & $2.0 \pm 0.8$ & $1.5 \pm 0.9$ & $2.3 \pm 1.0$ \\
\hline
\end{tabular}

\section{Discussione}

Il trattamento dialitico ideale in corso di IRA dovrebbe avere come obiettivo quello di essere adeguato al PCR, permettere una buona correzione dell'acidosi metabolica e del bilancio elettrolitico, buona tollerabiltà, stabilità cardiovascolare nonostante un'adeguata rimozione di liquidi, ridotto o assente uso di eparina. Le tecniche dialitiche utilizzate per il trattamento dell'IRA sono sia la dialisi peritoneale che la dialisi extracorporea.
Quest'ultima è da preferire per la maggiore rapidità d' azione e per le numerose possibilità di personalizzare il trattamento. Inoltre, alcuni Autori $(10,11)$ hanno recentemente riportato che l'uso di membrane altamente biocompatibili potrebbe incidere sulla prognosi dei pazienti affetti da IRA in trattamento dialitico, migliorandola. Nella nostra esperienza, la sopravvivenza media è risultata essere del $55.2 \%$, con una differenza altamente significativa tra il gruppo affetto da IRA non complicata e il gruppo 
con MOF (rispettivamente $80 \%$ e $35 \%$ ). Questo dato conferma l'importanza della MOF come fattore che influenza pesantemente la prognosi di tali pazienti. Al contrario, i nostri dati mostrano come l'uso di differenti metodiche, mentre migliora la tollerabilità del trattamento, non incide sulla sopravvivenza, specialmente nei pazienti critici. Inoltre l'uso di membrane biocompatibili, da noi utilizzate in tutti i casi oggetto dello studio, non ha inciso sulla mortalità, ma, in accordo con altri Autori (12), migliora la stabilità cardiovascolare che rappresenta ancor'oggi uno dei maggiori problemi del trattamento dialitico in corso di IRA, specie se complicata da MOF. Tra le tecniche da noi utilizzate, l'uso di bicarbonato dialisi standard e di AFB è di grande affidabilità nei casi di IRA non complicata. Inoltre, la AFB ha una migliore tollerabilità per la possibilità di personalizzare il trattamento, adattandolo sulle diverse necessità cliniche. L'emofiltrazione continua sembra essere la metodica di scelta nei pazienti critici con MOF (13). Infatti sia la CAVH che la CVVH, con la possibilità di utilizzare anche il bagno di dialisi, assicurano la possibilità di una buona depurazione, una adeguata rimozione di liquidi ed una tollerabilità ottimale. La CVVH rispetto alla CAVH, pur nei limiti numerici della nostra esperienza, presenta il vantaggio di poter programmare il calo ponderale, evita di dover pungere l'arteria femorale, e consente l'uso di una cannula bilume riducendo i rischi infettivi. Come svantaggi possiamo segnalare soltanto problemi di natura logistico-organizzativa. L'uso della Daily-HD in pazienti critici affetti da IRA in corso di MOF deve essere limitato ai casi che presentano gravi problemi di coagulazione.

In conclusione possiamo affermare che l'IRA è una sindrome la cui prognosi è severamente condizionata dalla presenza di insufficienza multiorgano. Infatti, quando compare in corso di Multiple Organ Failure ha una prognosi estremamente grave. In questo caso il trattamento dialitico può essere praticato con varie metodiche che presentano una diversa tollerabilità, assicurano in tutti i casi un sufficiente grado di depurazione, un adeguato bilancio idroelettrolitico, ma non sembrano incidere sulla prognosi di tali pazienti.

\section{BIBLIOGRAFIA}

1. Spiegel DM, Ullian ME, Zerbe GO, Berl T. Determinant of survival and recovery in acute renal failure patients dialyzed in intensive care units. Am J Nephrol 1991; 11: 44-7.

2. Biesenbach G, Zazgornik J, Kaiser W, Grafinger P, Stuby U, Necek S. Improvement in prognosis of patients with acute renal failure over a period of 15 years: An analysis of 710 cases in dialysis center. Am J Nephrol 1992; 12: 319-25.

3. Meloni C, Morosetti M, Meschini L. Blood purification for acute renal failure: convenient strategy related to clinical conditions. Blood Purif 1996; 14: 242-8.

4. Sandroni S, Arora N, Powell N. Performance characteristics of contemporary hemodialysis and venovenous hemofiltration in acute renal failure. Renal Fail 1992; 14: 571-4.

5. Meloni C, Morosetti M, Meschini L, Taccone-Gallucci M, Casciani CU. Acetate-free biofiltration in acute renal failure. Artif Organs 1993; 17: 188-90.

6. Gotch FA. Kinetic modeling in hemodialysis. In: Nissenson A, Gentile D, Fine RA eds. Clinical Dialysis. Norwalk: Appleton-CenturyCrofts, 1990; 118-46.

7. Knaus WA, Draper EA, Douglas PW, Zimmerman JE. Apache II: A severity of disease classification system. Crit Care Med 1985; 13: 818-29.

8. Buoncristiani U, Quintiliani G, Cozzari M, Giombini L, Ragaiolo M. Daily dialysis: Long-term clinical metabolic results. Kidney Int 1988; 33 (suppl): 137-40.

9. Golper TA, Price J. Continuous venovenous hemofiltration for acute renal failure in the intensive care setting. Technical considerations. ASAIO J 1994; 40 (4): 936-9.

10. Hakim RM, Wingard RL,
Parker RA. Effect of the dialysis membrane in the treatment of patients with acute renal failure. $\mathrm{N}$ Engl J Med 1994; 331: 1338-42.

11. Turney JH. Acute renal failure: Some progress? N Engl J Med 1994; 333: 1372-4.

12. Davenport A, Davison AM, Will EJ. Membrane biocompatibility: Effects on cardiovascular stability in patients on hemofiltration. Kidney Int 1993; 43(suppl): 230-4.

13. Bellomo R, Farmer M, Boyce N. A prospective study of continuous hemodiafiltration in the management of severe renal failure in critically ill surgical patients. Renal Fail 1994; 16 (6): 759-66. 